\title{
Molecular mechanism of double Holliday junction dissolution
}

\author{
Paolo Swuec and Alessandro Costa*
}

\begin{abstract}
Processing of homologous recombination intermediates is tightly coordinated to ensure that chromosomal integrity is maintained and tumorigenesis avoided. Decatenation of double Holliday junctions, for example, is catalysed by two enzymes that work in tight coordination and belong to the same 'dissolvasome' complex. Within the dissolvasome, the RecQ-like BLM helicase provides the translocase function for Holliday junction migration, while the topoisomerase III alpha-RMI1 subcomplex works as a proficient DNA decatenase, together resulting in double-Holliday-junction unlinking. Here, we review the available architectural and biochemical knowledge on the dissolvasome machinery, with a focus on the structural interplay between its components.
\end{abstract}

\section{Introduction}

RecQ helicases play a key role in genome stability maintenance. In humans, five distinct proteins containing a RecQ-like module are involved in a set of diverse nucleic acid transactions, including DNA replication, recombination and repair. Three of these proteins (the Werner syndrome helicase WRN, the Bloom's syndrome protein BLM and RecQL4) are found mutated in rare genetic disorders, characterised by chromosomal aberrations that are in turn associated with cancer predisposition and premature aging [1]. Mutations in the BLM protein, for example, cause Bloom's syndrome [2], whose hallmark is a pronounced increase in sister chromatid exchange [3].

The dissolvasome complex collapses and unlinks a double Holliday junction

DNA double-strand breaks can be repaired by homologous recombination, whose key intermediate is the fourway Holliday junction. During meiosis, Holliday junctions are cut by endonucleases to generate crossovers that are key to the proper segregation of chromosomes $[4,5]$. In somatic cells crossovers may cause deleterious mutations and are suppressed by BLM [6]. In a process aided by topoisomerases [7-9], BLM catalyses the convergent migration of a double Holliday junction $(\mathrm{dHJ})$, collapsing it

\footnotetext{
* Correspondence: alessandro.costa@cancer.org.uk

Clare Hall laboratories, Cancer Research U.K. London Research Institute, London EN6 3LD, UK
}

into a hemicatenane [10]. This intermediate is unlinked by Topoisomerase III $\alpha$ (TopoIII $\alpha)$ to generate non-crossover products [6]. BLM and TopoIII $\alpha$ work in the context of a hetero-complex named the dissolvasome [10] that also includes the "RecQ-mediated instability factors", RMI1 and RMI2 [11,12]. The interaction and concerted activities of the BLM and TopoIII $\alpha$ complex is sufficient to drive $\mathrm{dHJ}$ dissolution [6] but RMI1 has been found to stimulate this reaction $[13,14]$. Although the molecular basis of $\mathrm{dHJ}$ dissolution remains unclear, a wealth of information is available on the structure and function of the isolated dissolvasome components and their subcomplexes.

\section{BLM catalyses the convergent migration of two Holliday junctions}

(Sos1 in yeast) provides the ATP-dependent motor n to convergently migrate a $\mathrm{dHJ}[15,16]$ and two separate domains (Figure 1A, [17]). The Ninal region comprises a partially unstructured nondomain (NTD), which is the target of diverse t-translational modifications $[18,19]$ and might contain o-oligomerisation module [20]. The BLM Cstranded DNA translocases that can function as mono(21]. In RecQ proteins, a highly conserved binding and contains a bipartite active site for ATP binding and hydrolysis, where catalytic residues are contributed by two fused, neighbouring RecA-type modules [22]. The RecQ C-terminal (RQC) domain contains a zinc 


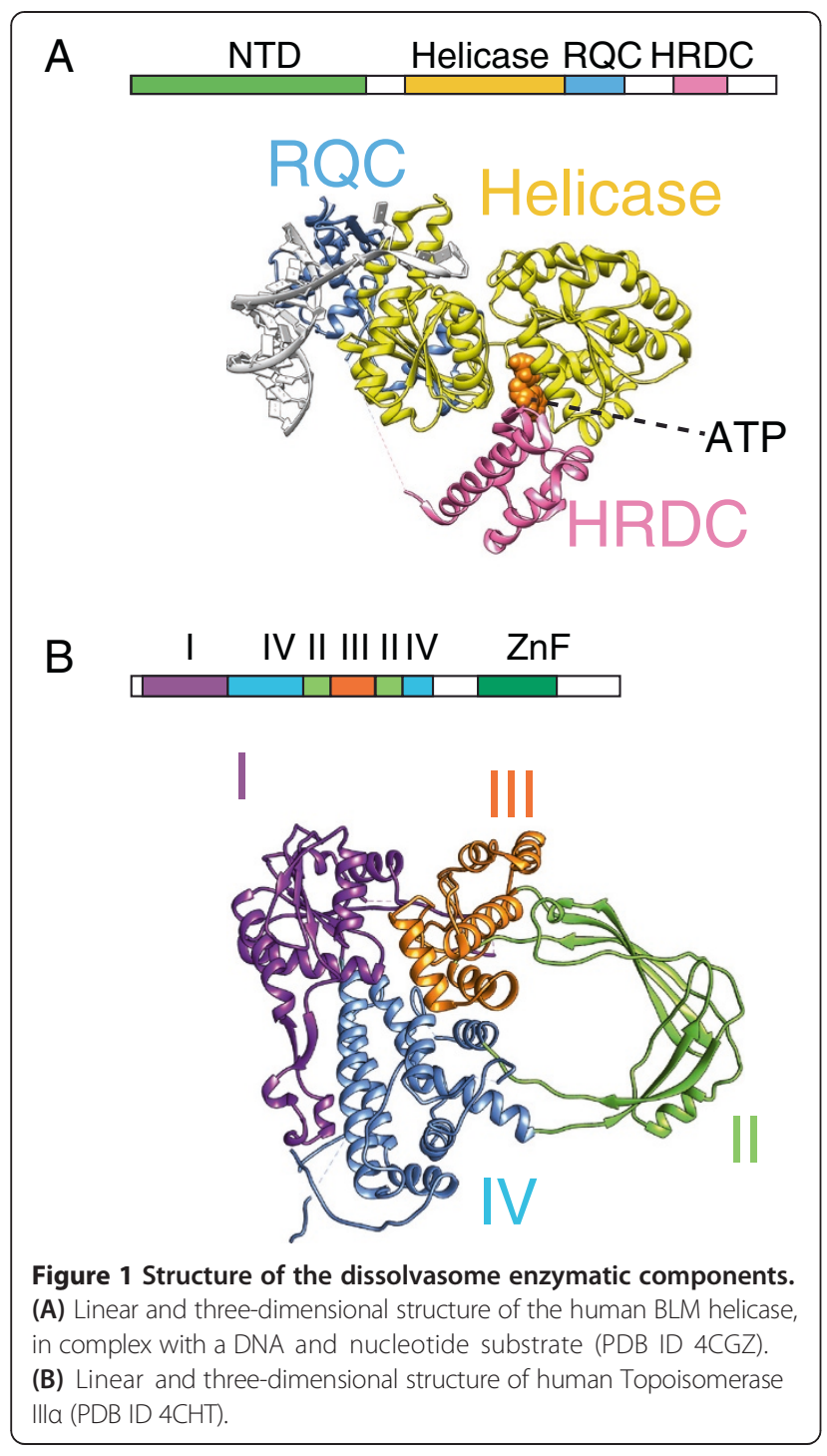

finger motif and a duplex-DNA binding Winged-Helix subdomain [22], which provides the proteinaceous pin to split the two DNA strands at the fork nexus (Figure 1A, PDB entry 4CGZ and [23]). Unexpectedly, a minimal helicase module containing the RecA sandwich and the zinc finger domain but lacking the Winged-Helix domain has been recently described, which can unwind a fork substrate in vitro [24]. Lastly, the Helicase and RNaseD C-terminal (HRDC) domain is a separate, globular entity that confers substrate specificity to BLM $[25,26]$, being required for Holliday junction dissolution or unwinding, but not for the unwinding of a simple DNA-fork substrate [27].

Various reports indicate that BLM can be found in distinct oligomeric states that co-exist in solution, ranging from monomers and dimers $[28,29]$ to tetramers and hexamers [30]. The effect of substrate binding of the
BLM oligomeric state is still a matter of debate and the functional implication of self-assembly is still unclear, however it might play a role in orienting multiple helicase motors for the convergent migration of two Holliday junctions during dissolution [17].

\section{Topoisomerase III alpha is structurally related to a type1 $\mathrm{A}$} relaxase

The second key player in the $\mathrm{dHJ}$ dissolution reaction is TopoIII $\alpha$, which unlinks a hemicatenane intermediate during the final step of dissolution [10]. TopoIII $\alpha$ belongs to the type1A class of topoisomerases, which are padlockshaped enzymes that effect changes in DNA topology in an ATPase independent manner [31,32]. Type 1A topoisomerases are indeed markedly distinct from the ATPdependent type-II topoisomerases and contain a 4-domain core (I-IV) that can bind, cleave and reseal single-stranded DNA substrates (Figure 1B, [33]). This process occurs through a transesterification reaction mediated by a nucleophilic tyrosine (Tyr337 in human TopoIII $)$, thus creating a transient 'DNA gate' for nucleic-acid strand passage (between domains I and III) [34]. Type1A topoisomerases can be classified into two groups: $i$ ) relaxases (such as the E. coli TopoI), which efficiently remove negative supercoils from a covalently closed plasmid [35] and ii) decatenases (e.g. E. coli TopoIII), which can unlink catenated DNA molecules [36]. Although E. coli TopoI and TopoIII overall share the same fold, TopoIII contains small additional elements, as for example a short domain IV insertion ('decatenation loop'), which lines the topoisomerase central cavity and is important for catenane unlinking (Figure 2A, [37,38]). Unexpectedly, TopoIII $\alpha$ lacks the decatenation loop and appears structurally more similar to type1A relaxases than to decatenases [31], raising the question of how TopollI $\alpha$-mediated hemi-catenane unlinking is achieved. Recent work indicates that RMI1 plays a key structural role in this process $[31,39]$.

\section{RMI1 stabilises the open form of the Topollla DNA gate}

RMI1 stimulates the double-Holliday junction dissolution reaction and directly interacts with both the BLM helicase and TopoIII $[13,14,40]$. It is composed of an N-terminal helical bundle followed by an oligonucleotide/oligosaccharide binding (OB) domain, which is connected, via a poorly conserved linker, to a higher-eukaryote specific second OB scaffold (Figure 2B, [41,42]). The second OB fold in turn contacts the related RMI2 factor (absent in yeast), together providing a docking site for other genome stability maintenance machineries [43,44]. NTD RMI1 is sufficient to stimulate $\mathrm{dHJ}$ dissolution [42,45] and in particular acts in the later stages of this reaction [7,39]. In fact, while Rmil has no effect on the initial rate of Holliday junction migration, as shown in yeast, it has an important role in 

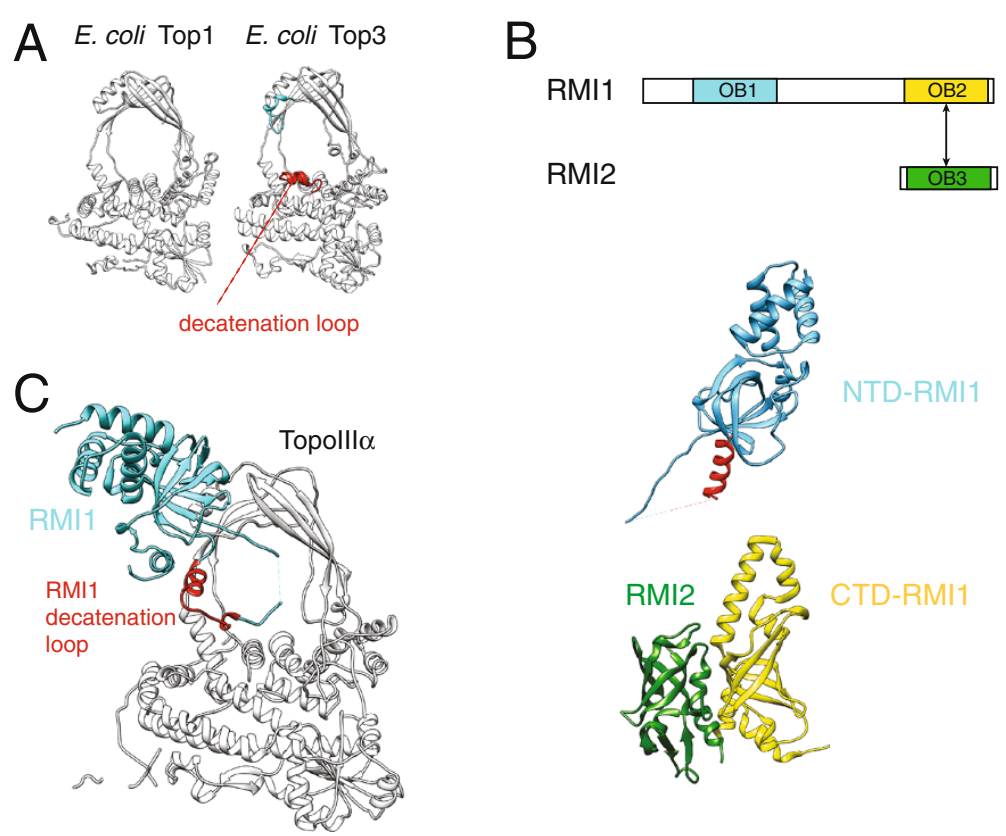

Figure 2 Topoisomerase IIIa and RMI1 reconstitute a DNA decatenase. (A) Structure of the E. coli Topol relaxase (PDB ID 1ECL) and the E. coli Topolll decatenase (PDB ID 1D6M). Topolll contains specific insertions lining the pore of the topoisomerase toroid which are critical for efficient decatenation. (B) Linear and three dimensional structure of RMI1 and RMI2 (PDB IDs 3NBI and 4DAY). The decatenation loop of RMI1 is highlighted in red. (C) The N-terminal domain of RMl1 contributes to Topollla a decatenation loop in trans (marked in red, PDB ID 4CGY).

removing the last linkages between two recombining DNA molecules [7]. Further work in yeast has shown that Rmi1 acts by stabilising the open form of the Top3 DNA gate (orthologous to TopoIII $\alpha$ ), hence slowing down the kinetics of DNA relaxation while favouring decatenation [39]. Recent crystallographic studies on the human TopoIII $\alpha$-RMI1 sub-complex from Nico Thomä's group elucidate the molecular basis of the RMI1 stimulatory role in decatenation. The NTD RMI1 OB fold docks onto TopoIII $\alpha$ domain II and contributes an insertion loop that lines the topoisomerase central cavity, while also reaching out to domain III at the DNA gate (Figure 2C, [31]). Close inspection of the TopoIII $\alpha$-RMI1 complex reveals that the RMI1 insertion loop indeed contributes topoisomeraseinteracting elements in regions where TopoIII $\alpha$ diverges from the E. coli TopoIII decatenase (Figure 2D, see previous paragraph). Hence, RMI1 appears to donate a DNA decatenation loop to TopoIII in trans, which could be sufficient to turn a relaxase into a decatenase. Although the crystal structure of TopoIII $\alpha$-RMI1 was solved in a closed configuration, the insertion loop appears poised to push the gate open, locking the topoisomerase in a covalently-attached, open, DNA-bound form that would promote DNA decatenation. Consistent with this model, a scrambled insertion-loop mutant, which does not interfere with complex formation, fails to stimulate $\mathrm{dHJ}$ dissolution, reverting yeast Top3 back to a modest decatenase [31].
BLM plays a structural role in modulating strand passage

How BLM structurally and functionally interacts with the TopoIII $\alpha-$ RMI1 sub-complex is still poorly understood. It is known that BLM contacts NTD RMI1 $[13,14,45]$ while TopoIII interacts with the NTD of BLM/Sgs1 $[46,47]$. In Drosophila, which appears to lack RMI1, a fly-specific Cterminal insertion in TopoIII $\alpha$ that contacts BLM at an unknown site, is essential for dissolution and might functionally substitute RMI1 [9]. It remains to be established whether the RecQ and Top1A catalytic domains ever come in direct contact during dissolution and whether any allosteric communication occurs between the two modules.

Similarly unclear is the nature of the BLM-TopoIII $\alpha$ catalytic interplay [48]. Work in Drosophila, for example, suggests that BLM and TopoIII $\alpha$ could coordinate their activities to achieve branch migration [8]. In vitro studies on this system indicate that, while the isolated BLM is incapable of migrating a topologically constrained $\mathrm{dHJ}$, vigorous migration is observed when Drosophila Top1 is added to the reaction mixture, although branches pause before merging into a hemicatenane. When BLM is instead assayed with TopoIII $\alpha$, the reaction goes to completion without any pausing, indicating that dissolution is indeed a highly processive reaction, and hinting at a possible catalytic interplay between the two enzymes [8].

Work in yeast, however, indicates that Sgs1/BLM has a role in modulating strand passage by TopoIII $\alpha$ and RMI1, 
which does not depend on the catalytic activity of the helicase module [39]. In a catenation assay on plasmids containing a preformed DNA bubble, Sgs1-Top3-Rmi1 appears proficient in converting individual DNA molecules into multimeric catenanes, irrespective of the presence of a wild type or a catalytically-dead mutant of Sgs1 [39]. These results indicate that Sgs1/BLM has a structural, rather than catalytic, role in promoting strand passage in the dissolvasome. It remains to be determined whether Sgs1/BLM binding acts by repositioning the decatenation loop insertion in RMI1 or rather contacts the topoisomerase gate directly, hence controlling the TopoIII $\alpha$ opening/ closure state.

\section{Concluding remarks}

Recent advances shed light on the role of isolated dissolvasome components in the convergent migration of $\mathrm{dHJ}$ junctions and hemi-catenane unlinking. Whether BLM and TopoIII $\alpha$-RMI1 allosterically influence their reciprocal catalytic activities still remains unclear. A comprehensive view of the molecular basis of $\mathrm{dHJ}$ dissolution will be likely achieved once the high-resolution structure of the full dissolvasome assembly is determined.

\section{Competing interests}

The authors declare that they have no competing interests.

\section{Authors' contributions}

PS and AC wrote the manuscript. Both authors read and approved the final manuscript.

\section{Acknowledgments}

We would like to thank Julian Gannon for reading the manuscript and members of the Costa laboratory for useful comments. A special thanks goes to James Berger, Stephen West, Andrew Deans and Adelina Davies for fruitful discussions. Work in the Costa lab is supported by Cancer Research UK.

Received: 8 February 2014 Accepted: 18 June 2014

Published: 9 July 2014

\section{References}

1. Chu WK, Hickson ID: RecQ helicases: multifunctional genome caretakers. Nat Rev Cancer 2009, 9:644-654.

2. Ellis NA, Groden J, Ye TZ, Straughen J, Lennon DJ, Ciocci S, Proytcheva M, German J: The Bloom's syndrome gene product is homologous to RecQ helicases. Cell 1995, 83:655-666.

3. Chaganti RS, Schonberg S, German J: A manyfold increase in sister chromatid exchanges in Bloom's syndrome lymphocytes. Proc Natl Acad Sci U S A 1974, 71:4508-4512.

4. Matos J, Blanco MG, Maslen S, Skehel JM, West SC: Regulatory control of the resolution of DNA recombination intermediates during meiosis and mitosis. Cell 2011, 147:158-172.

5. Zakharyevich K, Tang S, Ma Y, Hunter N: Delineation of joint molecule resolution pathways in meiosis identifies a crossover-specific resolvase. Cell 2012, 149:334-347.

6. Wu L, Hickson ID: The Bloom's syndrome helicase suppresses crossing over during homologous recombination. Nature 2003, 426:870-874.

7. Cejka P, Plank JL, Bachrati CZ, Hickson ID, Kowalczykowski SC: Rmi1 stimulates decatenation of double Holliday junctions during dissolution by Sgs1-Top3. Nat Struct Mol Biol 2010, 17:1377-1382.

8. Chen SH, Plank JL, Willcox S, Griffith JD, Hsieh TS: Top3alpha is required during the convergent migration step of double Holliday junction dissolution. PLoS One 2014, 9:e83582.
9. Chen SH, Wu CH, Plank JL, Hsieh TS: Essential functions of C terminus of Drosophila Topoisomerase Illalpha in double holliday junction dissolution. J Biol Chem 2012, 287:19346-19353.

10. Mankouri HW, Hickson ID: The RecQ helicase-topoisomerase III-Rmi1 complex: a DNA structure-specific 'dissolvasome'? Trends Biochem Sci 2007, 32:538-546.

11. Singh TR, Ali AM, Busygina V, Raynard S, Fan Q, Du CH, Andreassen PR, Sung P, Meetei AR: BLAP18/RMI2, a novel OB-fold-containing protein, is an essential component of the Bloom helicase-double Holliday junction dissolvasome. Genes Dev 2008, 22:2856-2868.

12. Yin J, Sobeck A, Xu C, Meetei AR, Hoatlin M, Li L, Wang W: BLAP75, an essential component of Bloom's syndrome protein complexes that maintain genome integrity. EMBO J 2005, 24:1465-1476.

13. Raynard S, Bussen W, Sung P: A double Holliday junction dissolvasome comprising BLM, topoisomerase Illalpha, and BLAP75. J Biol Chem 2006, 281:13861-13864.

14. Wu L, Bachrati CZ, Ou J, Xu C, Yin J, Chang M, Wang W, Li L, Brown GW, Hickson ID: BLAP75/RMI1 promotes the BLM-dependent dissolution of homologous recombination intermediates. Proc Natl Acad Sci U S A 2006, 103:4068-4073.

15. Cejka P, Kowalczykowski SC: The full-length Saccharomyces cerevisiae Sgs1 protein is a vigorous DNA helicase that preferentially unwinds holliday junctions. J Biol Chem 2010, 285:8290-8301.

16. Plank JL, Wu J, Hsieh TS: Topoisomerase Illalpha and Bloom's helicase can resolve a mobile double Holliday junction substrate through convergent branch migration. Proc Natl Acad Sci U S A 2006, 103:11118-11123.

17. Manthei KA, Keck JL: The BLM dissolvasome in DNA replication and repair. Cell Mol Life Sci 2013, 70:4067-4084.

18. Rao VA, Fan AM, Meng L, Doe CF, North PS, Hickson ID, Pommier Y: Phosphorylation of BLM, dissociation from topoisomerase Illalpha, and colocalization with gamma-H2AX after topoisomerase l-induced replication damage. Mol Cell Biol 2005, 25:8925-8937.

19. Wang J, Chen J, Gong Z: TopBP1 controls BLM protein level to maintain genome stability. Mol Cell 2013, 52:667-678.

20. Beresten SF, Stan R, van Brabant AJ, Ye T, Naureckiene S, Ellis NA: Purification of overexpressed hexahistidine-tagged BLM N431 as oligomeric complexes. Protein Expr Purif 1999, 17:239-248.

21. Singleton MR, Dillingham MS, Wigley DB: Structure and Mechanism of Helicases and Nucleic Acid Translocases. Annu Rev Biochem 2007, 76:23-50.

22. Bernstein DA, Zittel MC, Keck JL: High-resolution structure of the E.coli RecQ helicase catalytic core. EMBO J 2003, 22:4910-4921.

23. Lucic B, Zhang Y, King O, Mendoza-Maldonado R, Berti M, Niesen FH, Burgess-Brown NA, Pike AC, Cooper CD, Gileadi O, Vindigni A: A prominent beta-hairpin structure in the winged-helix domain of RECQ1 is required for DNA unwinding and oligomer formation. Nucleic Acids Res 2011, 39:1703-1717

24. Gyimesi M, Harami GM, Sarlos K, Hazai E, Bikadi Z, Kovacs M: Complex activities of the human Bloom's syndrome helicase are encoded in a core region comprising the RecA and Zn-binding domains. Nucleic Acids Res 2012, 40:3952-3963.

25. Killoran MP, Keck JL: Structure and function of the regulatory C-terminal HRDC domain from Deinococcus radiodurans RecQ. Nucleic Acids Res 2008, 36:3139-3149.

26. Kim YM, Choi BS: Structure and function of the regulatory HRDC domain from human Bloom syndrome protein. Nucleic Acids Res 2010, 38:7764-7777.

27. Wu L, Chan KL, Ralf C, Bernstein DA, Garcia PL, Bohr VA, Vindigni A, Janscak $P$, Keck JL, Hickson ID: The HRDC domain of BLM is required for the dissolution of double Holliday junctions. EMBO J 2005, 24:2679-2687.

28. Gyimesi M, Pires RH, Billington N, Sarlos K, Kocsis ZS, Modos K, Kellermayer MS, Kovacs M: Visualization of human Bloom's syndrome helicase molecules bound to homologous recombination intermediates. FASEB $\lrcorner$ 2013, 27:4954-4964.

29. Xu YN, Bazeille N, Ding XY, Lu XM, Wang PY, Bugnard E, Grondin V, Dou SX, Xi XG: Multimeric BLM is dissociated upon ATP hydrolysis and functions as monomers in resolving DNA structures. Nucleic Acids Res 2012, 40:9802-9814

30. Karow JK, Newman RH, Freemont PS, Hickson ID: Oligomeric ring structure of the Bloom's syndrome helicase. Curr Biol 1999, 9:597-600.

31. Bocquet NBAH, AAbdulrahman W, Larsen NB, Mahamadou F, Cavadini S, Bunker RD, Kowalczykowski SC, Cejka P, Jockspm ID, Thoma NH: Structural 
and mechanistic insight into Holliday junction dissolution by

Topoisomerase III alpha and RMI1. Nat Struct Mol Biol 2014, 21(3):261-268.

32. Goulaouic H, Roulon T, Flamand O, Grondard L, Lavelle F, Riou JF:

Purification and characterization of human DNA topoisomerase IIlalpha. Nucleic Acids Res 1999, 27:2443-2450.

33. Lima CD, Wang JC, Mondragon A: Three-dimensional structure of the $67 \mathrm{~K} \mathrm{~N}$-terminal fragment of E. coli DNA topoisomerase I. Nature 1994 367:138-146.

34. Feinberg H, Lima CD, Mondragon A: Conformational changes in E. coli DNA topoisomerase I. Nat Struct Biol 1999, 6:918-922.

35. Wang JC: Interaction between DNA and an Escherichia coli protein omega. J Mol Biol 1971, 55:523-533.

36. Nurse $\mathrm{P}$, Levine $\mathrm{C}$, Hassing $\mathrm{H}$, Marians $\mathrm{KJ}$ : Topoisomerase III can serve as the cellular decatenase in Escherichia coli. J Biol Chem 2003, 278:8653-8660.

37. Li Z, Mondragon A, Hiasa H, Marians KJ, DiGate RJ: Identification of a unique domain essential for Escherichia coli DNA topoisomerase III-catalysed decatenation of replication intermediates. Mol Microbiol 2000, 35:888-895

38. Mondragon A, DiGate R: The structure of Escherichia coli DNA topoisomerase III. Structure 1999, 7:1373-1383.

39. Cejka P, Plank JL, Dombrowski CC, Kowalczykowski SC: Decatenation of DNA by the S. cerevisiae Sgs1-Top3-Rmi1 and RPA Complex: A Mechanism for Disentangling Chromosomes. Mol Cell 2012, 47:886-896.

40. Kennedy JA, Daughdrill GW, Schmidt KH: A transient alpha-helical molecular recognition element in the disordered $\mathrm{N}$-terminus of the Sgs 1 helicase is critical for chromosome stability and binding of Top3/Rmi1. Nucleic Acids Res 2013, 41:10215-10227.

41. Hoadley KA, Xu D, Xue Y, Satyshur KA, Wang W, Keck JL: Structure and cellular roles of the RMI core complex from the bloom syndrome dissolvasome. Structure 2010, 18:1149-1158.

42. Wang F, Yang Y, Singh TR, Busygina V, Guo R, Wan K, Wang W, Sung P, Meetei AR, Lei M: Crystal structures of RMI1 and RMI2, two OB-fold regulatory subunits of the BLM complex. Structure 2010, 18:1159-1170.

43. Deans AJ, West SC: FANCM connects the genome instability disorders Bloom's Syndrome and Fanconi Anemia. Mol Cell 2009, 36:943-953.

44. Hoadley KA, Xue Y, Ling C, Takata M, Wang W, Keck JL: Defining the molecular interface that connects the Fanconi anemia protein FANCM to the Bloom syndrome dissolvasome. Proc Natl Acad Sci U S A 2012, 109:4437-4442.

45. Raynard S, Zhao W, Bussen W, Lu L, Ding YY, Busygina V, Meetei AR, Sung P: Functional role of BLAP75 in BLM-topoisomerase Illalpha-dependent holliday junction processing. J Biol Chem 2008, 283:15701-15708.

46. Bennett RJ, Noirot-Gros MF, Wang JC: Interaction between yeast sgs1 helicase and DNA topoisomerase III. J Biol Chem 2000, 275:26898-26905.

47. Wu L, Davies SL, North PS, Goulaouic H, Riou JF, Turley H, Gatter KC, Hickson ID: The Bloom's syndrome gene product interacts with topoisomerase III. J Biol Chem 2000, 275:9636-9644.

48. Plank J, Hsieh TS: Helicase-appended topoisomerases: new insight into the mechanism of directional strand transfer. J Biol Chem 2009, 284:30737-30741.

doi:10.1186/2045-3701-4-36

Cite this article as: Swuec and Costa: Molecular mechanism of double Holliday junction dissolution. Cell \& Bioscience 2014 4:36.

\section{Submit your next manuscript to BioMed Central and take full advantage of:}

- Convenient online submission

- Thorough peer review

- No space constraints or color figure charges

- Immediate publication on acceptance

- Inclusion in PubMed, CAS, Scopus and Google Scholar

- Research which is freely available for redistribution 